\title{
Pediatric Adverse Drug Event Occurrence in a Community Hospital
}

\author{
Wendy L. Mosiman \\ Faculty: Elaine Steinke, J. Bryan Mann
}

Department of Nursing

\begin{abstract}
Hospitalized children are at greater risk for adverse drug events (ADEs) due to varying size, development and lack of pediatric specific dosing. The project determined ADE rate for40 randomly selected pediatric hospitalized inpatients utilizing the Pediatric-Focused Trigger Tool in a retrospective review. 171 triggers revealed 10 unique ADEs involving 8 patients, including ICU, general unit, and ER. Mean ADE rates were 25/100, 47.84/1000 days and 28.01/1000 medication doses, 50\% preventable. Constipation and oversedation werecommon. Opiates and benzodiazepines/anti-epilepsy medications were associated with ADEs. Thirty percent required initial or prolonged hospitalization. ADE rates in a community hospital were higher than the rates reported for children's hospitals. This rate will serve as a benchmark as new safety measures are implemented.
\end{abstract}

\section{Introduction}

Hospitalized children are at greater risk for adverse drug events (ADEs) due to varying size, development and lack of pediatric specific dosing and dosage forms. Little is known about the rate of ADEs in community hospitals. The purpose of this project was to determine the ADE rate among children admitted to a community hospital utilizing the Pediatric-Focused Trigger Tool [1]. Reason'stheory of error was used to frame the project and to determine potential causes of harm [2].

\section{Experiment, Results, Discussion, and Significance}

The prevalence of publicized medication errors led to the publication of a Sentinel Event Alert by The Joint Commission. This important program calls on all providers caring for pediatric patients to make changes to prevent medication-related harm. A variety of efforts to reduce the risk of medication errors and resultant harm have been studied. Adverse drug events are more likely to inspire changes in practice rather than meaningless error rates, not correlated with specific harm to a patient. Trigger tools have been found to find a 50 fold increase in ADE when compared to traditional reporting methods such as incident reports [3]. Trigger tools are the most efficient and cost effective method of obtaining information about ADEs [3,1].

Forty children from a Midwestern community hospital were randomly selected for retrospective chart review. The type of error, process problem, preventability, outcome and intervention was collected for all ADEs identified by triggers. Only ADEs verified by a second reviewer, a pediatric pharmacist, were included. Data were analyzed using confidence intervals, exact binomial distribution for proportions, Poisson distribution for rates, and proportion comparison using Chi square with Yeat's correction. A total of 171 triggers (4.28 per patient) and 10 unique ADEs involving eight patients were revealed. Mean ADE rates were 25 per 100 patients, 47.84 per 1000 patient days and 28.01 per 1000 medication doses. Fifty percent were determined to be preventable. Constipation and oversedation were most common ADEs. Opiates and benzodiazepines/antiepilepsy medications were commonly associated with ADEs. Event occurrence included: three patients in PICU (4 events); four patients on the general pediatric unit (4 events); and two infants required intubation after seizure treatment in the Emergency Department. Temporary harm occurred in $70 \%$ of patients and $30 \%$ required initial/prolonged hospitalization. The prescribing/ordering medication process stage was most frequently associated with harm.

\section{Conclusions}

This study identified $10 \mathrm{ADE}$, occurring in 8 patients, for overall rates of $25 \mathrm{ADE}$ per 100 patients, 47.84 ADE per 1000 patient days, 28.01 ADE per 1000 medications and 4.68 ADE per 1000 doses. One in five patients in this 
sample had an ADE. While none of these ADE were life threatening, the goal for all pediatric mediation delivery is to increase safety and decrease harm.

Of the preventable ADE discovered, some solutions exist to decrease patient harm. Constipation from opiates was the most common ADE. One patient was an oncology patient; the other three were orthopedic patients. Opioid induced constipation is related to the inhibition of gastric emptying, decreased rate of intestinal transit, and decrease in gut fluid reabsorption. While constipation may be considered by some a non significant adverse event, it causes discomfort and pain from abdominal distention, bloating, and hard stool in the rectum and can be severe enough to require additional hospital days [4].Sharek and others reported on an initiative involving forty two children's hospitals to decrease opioid related ADE [5]. They found rates of 33-51\% opioid related ADE. Constipation rates were decreased at these children's hospitals by the initiation of a "change package" which included recommending proactive routine stool softeners and laxatives when opiates are utilized. Treatment after constipation occurs is less effective than prophylactic treatment [6]. A similar opportunity exists to decrease constipation ADE in the non children's hospital pediatric patients by adopting this practice and adding a laxative and/or stool softener order to pediatric trauma and individual orthopedic surgeon pre printed order sets. If computerized order entry is available, a weight appropriate dosage may be automatically suggested to reduce omission of this important preventative treatment [7].

The other preventable ADE found by trigger tool was a medication level in excess of the target serum concentration. The result was present on the paper chart for more than 48 hours before the medication was stopped. A barcode medication administration system was implemented in September of 2009 in the community hospital. It contains a laboratory section that shows serum concentration results which may help to notify nurses of a non therapeutic level prior to administration of the medication. The follow up of this study, planned for March of 2010 will document ADE after implementation of both a smart pump system and barcode medication administration.

When compared to the Takata study of 960 children hospitalized in 12 children's hospital, the non-children's hospital group had an older population, shorter LOS, and fewer medications and doses per patient.

\section{Acknowledgements}

The author appreciates the advice and information received from CHCA staff member Tina Logsdon and Via Christi Hospital statistician Teresa Jones. Lela Fung, PharmD, Pediatric Specialist served as the second reviewer and provided invaluable insight.

[1] G.S. Takata, W. Mason, C. Taketomo, T. Logsdon and P.J. Sharek. Development, testing, and findings of a pediatric-focused trigger tool to identify medication-related harm in US children's hospitals. Pediatrics, 121(4), e927-935, 2008.

[2] J. Reason. Human Error.Cambridge, Mass: CambridgeUniversity Press, 1990.

[3] M.R. Cohen (Ed.). Medication Errors.Washington, DC: American Pharmacists Association, 2006.

[4] Y.S. Choi and J.A. Billings. Opioid antagonists: A review of their role in palliative care, focusing on use in opioid-related constipation. Journal of Pain Symptom Management, 24(1), 71-90, 2002.

[5] P.J. Sharek, R.E. McCleadJr, C. Taketomo, J.W. Luria, G.S. Takata, S. Glenn, B. Walti, M. Tanski, C. Nelson, T. R. Logsdon, C. Thurm and F. Federico. An Intervention to Decrease Narcotic-Related Adverse Drug Events in Children's Hospitals. Pediatrics 122: e861-e866, 2008.

[6[ M.J. Hockenberry. Wong's Essentials of Pediatric Nursing.St.Louis, MO: Elsevier Mosby, 2005.

[7] G.J. Kuperman and R.F. Gibson. Computer physician order entry: benefits, costs, and issues. Annals of Internal Medicine, 139, 31-39, 2003. 